\author{
Machine Learning \\ CS 4780/5780 - Fall 20I2 \\ Cornell University \\ Department of Computer Science
}

\title{
Recommendation Systems
}




\section{Antonio Bahamonde \\ Centro de Inteligencia Artificial. \\ Universidad de Oviedo at Gijón. Spain}

Spanish Association for Artificial Intelligence

From $9 / 2012$ till $7 / 2013$

Visiting Professor in Cornell University 


\section{Contents}

- Definitions

- Netflix Prize

- Similarity based methods

- Matrix factorization

- References 


\section{RS: Definitions}

RS help to match users with items

- Ease information overload

- Sales assistance (guidance, advisory, persuasion,...)

RS are software agents that elicit the interests and preferences of individual consumers [...] and make recommendations accordingly.

Different system designs / paradigms

- Based on availability of exploitable data

- Implicit and explicit user feedback

- Domain characteristics 


\section{Purpose and success criteria}

Retrieval perspective

- Reduce search costs

- Provide "correct" proposals - Users know in advance what they want

Recommendation perspective

- Serendipity- identify items from the Long Tail -Users did not know about existence
Recommend items

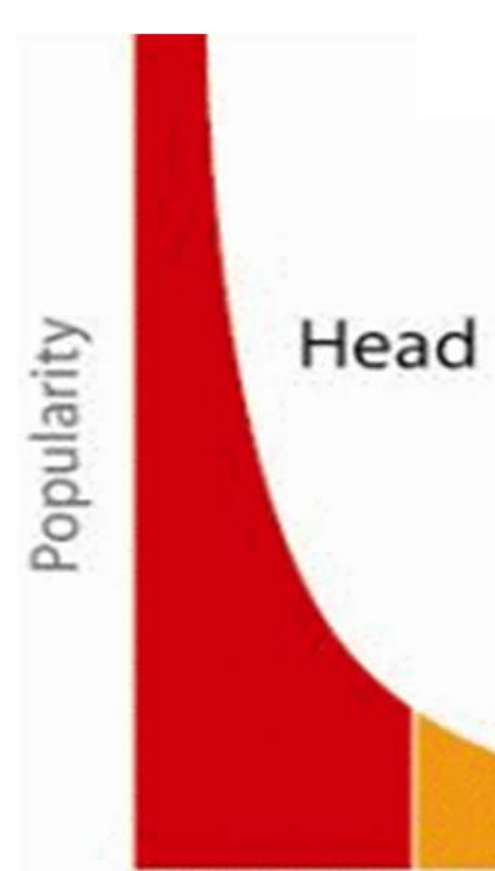

\author{
from the long tail
}

Long Tail

\section{Products}




\section{Purpose and success criteria}

Prediction perspective

- Predict to what degree users like an item

- Most popular evaluation scenario in research

Interaction perspective

- Give users a "good feeling" - Educate users about the product domain

- Convince/persuade users - explain

Finally, commercial perspective

- Increase "hit", "clickthrough", "lookers to bookers" rates

- Optimize sales margins and profit 


\section{Popular RS}

- Google

- Genius (Apple)

- last.fm
- Amazon

- Netflix

- TiVo 


\section{Popular RS:Waze}
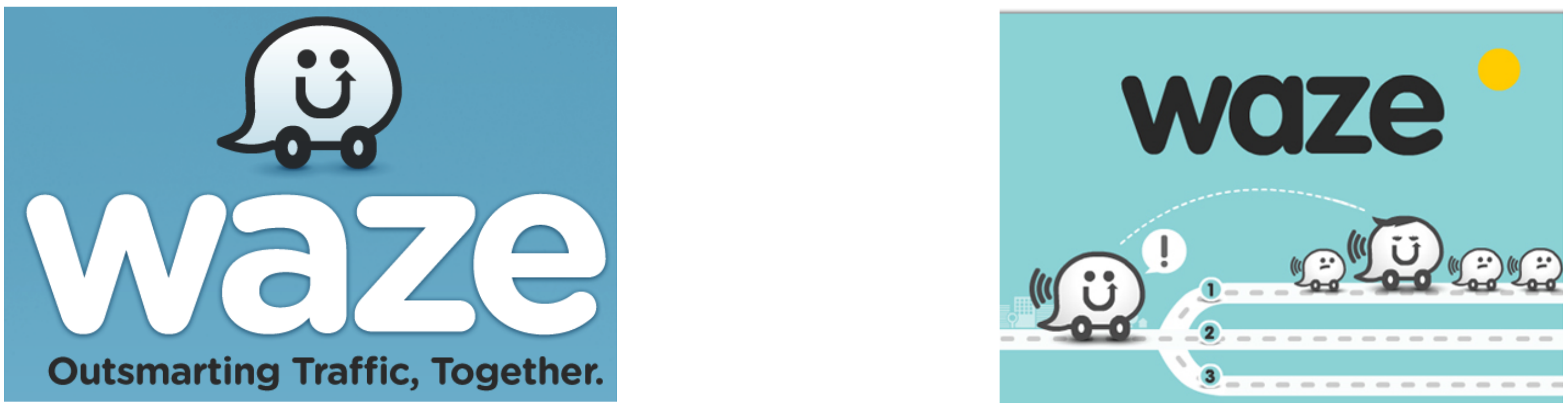

Now in the first line of actuality since Apple suggested its use instead of the failed new maps in iOS6. 


\section{Popular RS \\ (in everyday life)}

- Bestseller lists

- Top 40 music lists

- The "recent returns" shelf at the library

- Unmarked but well-used paths thru the woods

- Most popular lists in newspapers

- Many weblogs

- "Read any good books lately?" 


\section{Collaborative Filtering}

The most prominent approach to RS

- used by large, commercial e-commerce sites

- well-understood, various algorithms and variations exist

- applicable in many domains (book, movies, DVDs, ..)

Approach

- use the "wisdom of the crowd" to recommend items (crowdsourcing)

Basic assumption and idea

- users give ratings to catalog items (implicitly or explicitly)

- customers who had similar tastes in the past, will have similar tastes in the future 


\section{Collaborative Filtering}

- Relate two fundamentally different entities: users and items

explicit feedback (ratings)

implicit (purchase or browsing history, search patterns, ...) sometimes items descriptions by feature (content based)

- Approaches:

neighborhood latent factor 


\section{Naïve Neighborhood approach}

- item-item

- user-user

\begin{tabular}{|c|c|c|c|c|c|}
\hline & item1 & item2 & item3 & item4 & item5 \\
\hline alice & 5 & 3 & 4 & 4 & $?$ \\
\hline user1 & 3 & 1 & 2 & 3 & 3 \\
\hline user2 & 4 & 3 & 4 & 3 & 5 \\
\hline user3 & 3 & 3 & 1 & 5 & 4 \\
\hline user4 & 1 & 5 & 5 & 2 & 1 \\
\hline
\end{tabular}

Compute similarity and then prediction 


\section{Naïve Neighborhood approach}

- user-user

\begin{tabular}{|c|c|c|c|c|c|}
\hline & item1 & item2 & item3 & item4 & item5 \\
\hline alice & 5 & 3 & 4 & 4 & $?$ \\
\hline user1 & 3 & 1 & 2 & 3 & 3 \\
\hline user2 & 4 & 3 & 4 & 3 & 5 \\
\hline user3 & 3 & 3 & 1 & 5 & 4 \\
\hline user4 & 1 & 5 & 5 & 2 & 1 \\
\hline
\end{tabular}




\section{Naïve Neighborhood approach}

- item-item

\begin{tabular}{|c|c|c|c|c|c|}
\cline { 4 - 5 } \multicolumn{4}{c}{} & \multicolumn{3}{|c|}{} \\
\hline & item1 & item2 & item3 & item4 & item5 \\
\hline alice & 5 & 3 & 4 & 4 & $?$ \\
\hline user1 & 3 & 1 & 2 & 3 & 3 \\
\hline user2 & 4 & 3 & 4 & 3 & 5 \\
\hline user3 & 3 & 3 & 1 & 5 & 4 \\
\hline user4 & 1 & 5 & 5 & 2 & 1 \\
\hline
\end{tabular}




\section{Naïve Neighborhood approach}

- How to measure similarities?

$$
\begin{gathered}
\text { - correlation } \quad \rho(\boldsymbol{a}, \boldsymbol{b}) \\
\text { • cosine } \quad \cos (\boldsymbol{a}, \boldsymbol{b})=\frac{\boldsymbol{a}, \boldsymbol{b}}{\|\boldsymbol{a}\|\|\boldsymbol{b}\|} \\
b(u, i)=\overline{r(u, \cdot)}+\frac{\sum_{u^{\prime} \neq u} \operatorname{sim}\left(u, u^{\prime}\right)\left(r\left(u^{\prime}, i\right)-\overline{r\left(u^{\prime}, \cdot\right)}\right)}{\sum_{u^{\prime} \neq u} \operatorname{sim}\left(u, u^{\prime}\right)}
\end{gathered}
$$

similar formula for item-item 


\section{Naïve Neighborhood approach}

user-user using 2 neighbors
\begin{tabular}{|c|c|c|c|c|c|c|c|}
\hline$r(u, \cdot)$ & $\rho($ alice,$u)$ & & item 1 & item 2 & item3 & item4 & item5 \\
\hline 4,00 & & alice & 5 & 3 & 4 & 4 & $?$ \\
\hline 2,25 & 0,853 & user1 & 3 & 1 & 2 & 3 & 3 \\
\hline 3,50 & 0,707 & user2 & 4 & 3 & 4 & 3 & 5 \\
\hline 3,00 & 0 & user3 & 3 & 3 & 1 & 5 & 4 \\
\hline 3,25 & $-0,792$ & user4 & 1 & 5 & 5 & 2 & 1 \\
\hline
\end{tabular}

$b($ alice, item 5$)=4+\frac{(3-2.25) * 0.853+(5-3.5) * 0.707}{0.853+0.707}=5.09$ 


\section{Naïve Neighborhood approach}

item-item

using 2 neighbors

\begin{tabular}{|c|c|c|c|c|c|}
\hline & item1 & item2 & item3 & item4 & item5 \\
\hline alice & 5 & 3 & 4 & 4 & $?$ \\
\hline user1 & 3 & 1 & 2 & 3 & 3 \\
\hline user2 & 4 & 3 & 4 & 3 & 5 \\
\hline user3 & 3 & 3 & 1 & 5 & 4 \\
\hline user4 & 1 & 5 & 5 & 2 & 1 \\
\hline$\rho(i t e m 5, i)$ & 0,969 & $-0,478$ & $-0,428$ & 0,582 & \\
\hline$r(\cdot, i)$ & 3,2 & 3,0 & 3,2 & 3,4 & 3,25 \\
\hline
\end{tabular}

$b($ alice, item 5$)=3.25+\frac{(5-3.2) * 0.969+(4-3.4) * 0.582}{0.969+0.582}=4.6$ 


\section{Naïve Neighborhood approach}

- Scalability

- user-user is a memory based method

- millions of users

- does not scale for most real-world scenarios 


\section{Naïve Neighborhood approach}

- item-item

- is model based

- models learned offline are stored for predictions at run-time

- allows explanations

- no cold start problem 


\section{Naïve Neighborhood approach}

- However in all cases

- not all neighbors should be taken into account (similarity thresholds)

- not all item are rated (co-rated)

- not involved the loss function 


\section{Netflix Prize}

\section{Ëhe Atew Hork Eimes (Sep, 21, 2009):}

\section{Netflix Awards \$1 Million Prize and Starts a New Contest}

[...]try to predict what movies particular customers would prefer

"Accurately predicting the movies Netflix members will love is a key component of our service," said Neil Hunt, chief product officer (Netflix)
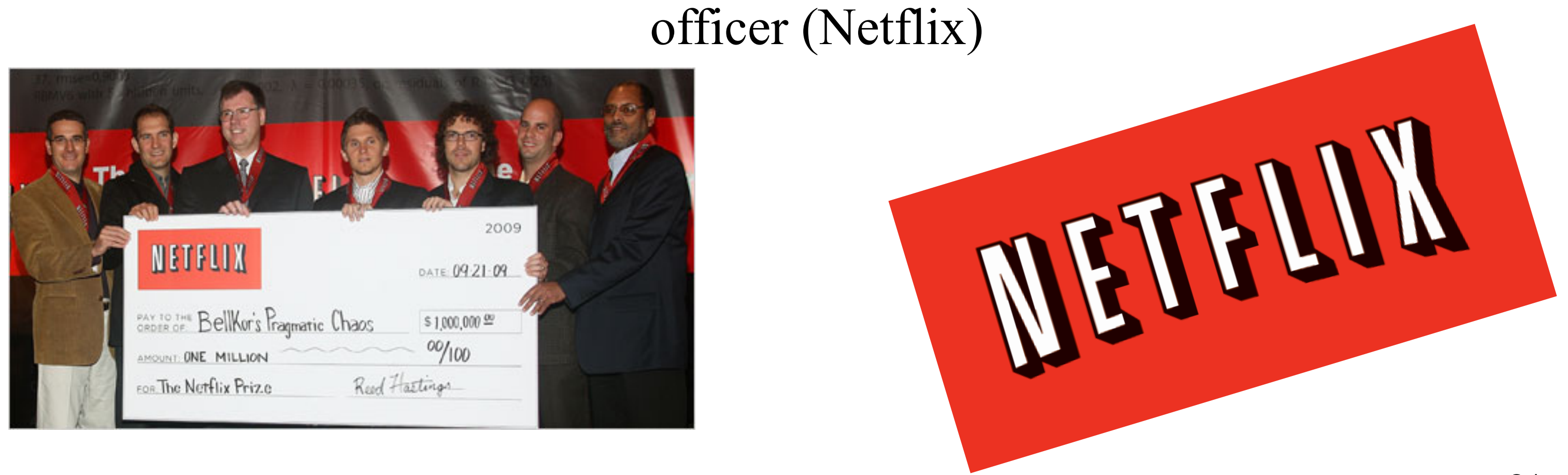


\title{
Netflix Prize
}

The Netflix dataset

\author{
more than 100 million movie ratings ( $1-5$ stars) \\ Nov II, I 999 and Dec 3I, 2005 \\ about 480, 189 users and $n=17,770$ movies \\ $99 \%$ of possible rating are missing \\ movie average 5600 ratings \\ user rates average 208 movies \\ training and quiz (test-prize) data
}




\section{Netflix Prize}

The loss function: root mean squared error (RMSE)

$$
R M S E=\sqrt{\frac{1}{|Q u i z|} \sum_{(u, i) \in Q u i z}(r(u, i)-b(u, i))^{2}}
$$

Netflix had its own system, Cinematch, which achieved $0.95 \mid 4$.

The prize was awarded to a system that reach RMSE below 0.8563 ( $10 \%$ improvement) 


\section{Netflix Prize}

- Leaderboard

\begin{tabular}{|l|l|c|c|c|}
\hline & Team & RMSE & Date & Hour \\
\hline 1 & BellKor's Pragmatic Chaos & 0,8567 & $26 / 07 / 09$ & $18: 18: 28$ \\
\hline 2 & The Emsemble & 0,8567 & $26 / 07 / 09$ & $18: 38: 22$ \\
\hline 3 & Grand Prize Team & 0,8582 & $10 / 07 / 09$ & $21: 24: 40$ \\
\hline 4 & $\begin{array}{l}\text { Opera Solutions and } \\
\text { Vandelay United }\end{array}$ & 0,8588 & $10 / 07 / 09$ & $01: 12: 31$ \\
\hline
\end{tabular}




\section{Netfilx prize winners}

Yehuda Koren, Robert M. Bell:Advances in Collaborative Filtering. Recommender Systems Handbook 20 I I: I45-I86

Yehuda Koren, Yahoo! Research

Robert Bell, $\quad$ AT\&T Labs - Research

Discuss similarity and matrix factorization approaches 


\section{Similarity approach revisited}

3 major components:

- data normalization

- neighbor selection

- determination of interpolation weights 


\section{Baseline approach}

Example:Titanic and Joe

- Average in Netflix: 3.7

- Joe critical: 0.3 less than average

- Titanic: 0.5 more than average (all users)

$$
\begin{aligned}
\mathrm{b}(\text { Joe, } \text { Titanic }) & =3.7-0.3+0.5=3.9 \\
b(u, i) & =\mu+\overline{b(u, \cdot)}+\overline{b(\cdot, i)} \\
b_{u, i} & =\mu+b_{u}+b_{i}
\end{aligned}
$$




\section{Baseline approach}

The equations sound appealing, but Koren and Bell propose to learn it using a least square approach:

$$
\min _{b_{*}} \sum_{(u, i) \in \mathscr{K}}\left(r_{u i}-\mu-b_{u}-b_{i}\right)^{2}+\lambda_{1}\left(\sum_{u} b_{u}^{2}+\sum_{i} b_{i}^{2}\right)
$$

where

$$
\mathscr{K}=\left\{(u, i) \mid r_{u i} \text { is known }\right\}
$$

and the last term is a regularization term to avoid overfitting 


\section{Baseline approach}

In the Netflix data

The average rating $(\mu)=3.6$

Learned bias

user $\left(b_{u}\right)$ has average 0.044

their absolute value $\left(\left|b_{\mathrm{u}}\right|\right)$ is 0.32

item (movie) $\left(b_{i}\right)$ has average -0.26 (s.d. 0.48 ),

$\left(\left|b_{i}\right|\right)$ is 0.43

\begin{tabular}{|c|c|c|c|}
\hline & baseline & Cinematch & Prize \\
\hline RMSE & 0,9799 & 0,9514 & 0,8567 \\
\hline
\end{tabular}




\section{Improvements}

- Koren \& Bell report significant improvements adding

- temporal dynamics (temporal drift of preferences)

- implicit feedback (movie rental history (not available; rated movies!),

\begin{tabular}{|c|c|c|c|c|c|}
\hline & baseline & + temporal & +tem (spline) & Cinematch & Prize \\
\hline RMSE & 0,9799 & 0,9771 & 0,9603 & 0,9514 & 0,8567 \\
\hline
\end{tabular}




\section{Matrix factorization}

- Tries to capture users and items relationships

- Based on well-known algebraic decomposition of matrices used before in Information Retrieval (LSI)

- Intended idea: consider latent variables

- As implemented by Koren and Bell, this approach won the Netflix Prize 


\section{Matrix factorization}

- Transform both items and users into a feature space of lower dimensionality $(k)$, the latent space

- Tries to explain ratings by characterizing both items and users on factors automatically learned from data.

- Factors might measure aspects as comedy, drama, amount of action, ...

- Efficient implementation offline

- Admit improvements in temporal drift and implicit feedback 


\section{Matrix factorization (SVD)}

SVD (singular value decomposition). Matrix $\mathrm{M}$ with rows users and columns items

$$
M=U * S *(\text { Items })^{\top}
$$

where

$$
\begin{gathered}
\mathrm{U}^{\top} \mathrm{U}=(\text { Items })^{\top}(\text { Items })=\mathbf{I} \\
S=\operatorname{diag}\left(s_{1}, \ldots, s_{n}\right), \quad s_{1} \geq s_{2}, \ldots s_{n-1} \geq s_{n} \geq 0 \\
\sqrt{S}=\operatorname{diag}\left(\sqrt{s_{1}}, \ldots, \sqrt{s_{n}}\right)
\end{gathered}
$$

and then

$$
M=(U * \sqrt{S}) *\left(\sqrt{S} * \text { Items }^{T}\right)
$$




\section{Matrix factorization (SVD)}

If we use only $\mathrm{k}$ dimensions

$$
M \cong M_{k}=U_{k^{*}} S_{k^{*}}(\text { Items })^{\top}
$$

This is a matrix of rank $\mathrm{k}$. The most similar to $\mathrm{M}$ with this rank.

$$
\begin{aligned}
& \mathrm{M}_{\mathrm{k}}=\text { \#Users } \\
& M_{k}=\left(U_{k} * \sqrt{S_{k}}\right) *\left(\sqrt{S_{k}} * \text { Items } s_{k}^{T}\right)
\end{aligned}
$$




\section{Matrix factorization}

Algorithm

$$
\begin{array}{ll}
\text { Let } M=r_{u i}-(\mu)-b_{u}-b_{i} ; & \% \text { fill missing values with } 0 \\
\text { [U S Items] }=\operatorname{svd}(M) ; & \% \text { fix } k<=\text { rank }(M) ; \\
\text { U_rep }=U_{k} * \text { Sqrt }\left(S_{k}\right) ; & \% \text { call pu row } u \text {-th of U_rep }
\end{array}
$$

Items_rep $=$ Items $s_{k}{ }^{*} \operatorname{sqrt}\left(S_{k}\right) ; \quad \%$ call qi row i-th of Items_rep

$$
\begin{gathered}
\hat{r}_{u i}=\mu+b_{i}+b_{u}+q_{i}^{T} p_{u} \\
M_{k}=\left(U_{k} * \sqrt{S_{k}}\right) *\left(\sqrt{S_{k}} * \text { Items } s_{k}^{T}\right)
\end{gathered}
$$




\section{Matrix factorization}

\section{In this case $\mathrm{M}$}

\begin{tabular}{|c|c|c|c|c|c|}
\hline & item1 & item2 & item3 & item4 & item5 \\
\hline alice & 5 & 3 & 4 & 4 & $?$ \\
\hline user1 & 3 & 1 & 2 & 3 & 3 \\
\hline user2 & 4 & 3 & 4 & 3 & 5 \\
\hline user3 & 3 & 3 & 1 & 5 & 4 \\
\hline user4 & 1 & 5 & 5 & 2 & 1 \\
\hline
\end{tabular}

\begin{tabular}{|c|}
\hline$b u$ \\
\hline 0,80 \\
\hline$-0,80$ \\
\hline 0,60 \\
\hline 0,00 \\
\hline$-0,40$ \\
\hline
\end{tabular}

\begin{tabular}{|c|c|c|c|c|c|}
\hline $\mathrm{b}_{\mathrm{i}}$ & 0,00 & $-0,20$ & 0,00 & 0,20 & 0,00 \\
\hline
\end{tabular}

\begin{tabular}{|l|l|}
\hline$\mu$ & 3,20 \\
\hline
\end{tabular}




\section{Matrix factorization}

$M=r_{u i}-(\mu)-b_{u}-b_{i} ; \quad \%$ fill missing values with 0

\begin{tabular}{|c|c|c|c|c|c|}
\hline & item1 & item2 & item3 & item4 & item5 \\
\hline alice & 1,0 & $-0,8$ & 0,0 & $-0,2$ & 0,0 \\
\hline user1 & 0,6 & $-1,2$ & $-0,4$ & 0,4 & 0,6 \\
\hline user2 & 0,2 & $-0,6$ & 0,2 & $-1,0$ & 1,2 \\
\hline user3 & $-0,2$ & 0,0 & $-2,2$ & 1,6 & 0,8 \\
\hline user4 & $-1,8$ & 2,4 & 2,2 & $-1,0$ & $-1,8$ \\
\hline
\end{tabular}




\section{Matrix factorization}

$M[U$ S Items $]=\operatorname{svd}(M) ;$

\begin{tabular}{|c|c|c|c|c|}
\hline \multirow{5}{*}{$U=$} & 0,348 & 0,445 & $-0,500$ & 0,640 \\
\hline & 0,185 & 0,148 & 0,842 & 0,389 \\
\hline & 0,478 & $-0,838$ & $-0,096$ & 0,226 \\
\hline & $-0,762$ & $-0,263$ & $-0,118$ & 0,414 \\
\hline & $-0,188$ & $-0,096$ & 0,136 & 0,465 \\
\hline \multirow{5}{*}{$S=$} & 0,000 & 0,000 & 0,000 & 0,000 \\
\hline & 2,591 & 0,000 & 0,000 & 0,000 \\
\hline & 0,000 & 1,258 & 0,000 & 0,000 \\
\hline & 0,000 & 0,000 & 0,440 & 0,000 \\
\hline & 0,000 & 0,000 & 0,000 & 0,000 \\
\hline
\end{tabular}




\section{Matrix factorization}

[U S Items] = svd(M);

Items $=$\begin{tabular}{|c|c|c|c|c|}
\hline$-0,359$ & 0,403 & 0,471 & $-0,536$ & $-0,447$ \\
\hline 0,515 & $-0,478$ & $-0,208$ & $-0,513$ & $-0,447$ \\
\hline 0,575 & 0,496 & 0,111 & 0,459 & $-0,447$ \\
\hline$-0,300$ & $-0,581$ & 0,385 & 0,474 & $-0,447$ \\
\hline$-0,431$ & 0,159 & $-0,758$ & 0,116 & $-0,447$ \\
\hline
\end{tabular}




\section{Matrix factorization}

For $\mathrm{k}=2$

$\bigcup_{k}=$
\begin{tabular}{|r|r|}
\hline$-0,143$ & 0,348 \\
\hline$-0,290$ & 0,185 \\
\hline$-0,097$ & 0,478 \\
\hline$-0,406$ & $-0,762$ \\
\hline 0,849 & $-0,188$ \\
\hline
\end{tabular}

$S_{k}=$
\begin{tabular}{|l|l|}
\hline 4,971 & 0,000 \\
\hline 0,000 & 2,591 \\
\hline 0,000 & 0,000 \\
\hline
\end{tabular}

Items $_{\mathrm{k}}=$

\begin{tabular}{|c|c|}
\hline$-0,359$ & 0,403 \\
\hline 0,515 & $-0,478$ \\
\hline 0,575 & 0,496 \\
\hline$-0,300$ & $-0,581$ \\
\hline$-0,431$ & 0,159 \\
\hline
\end{tabular}




\section{Matrix factorization}

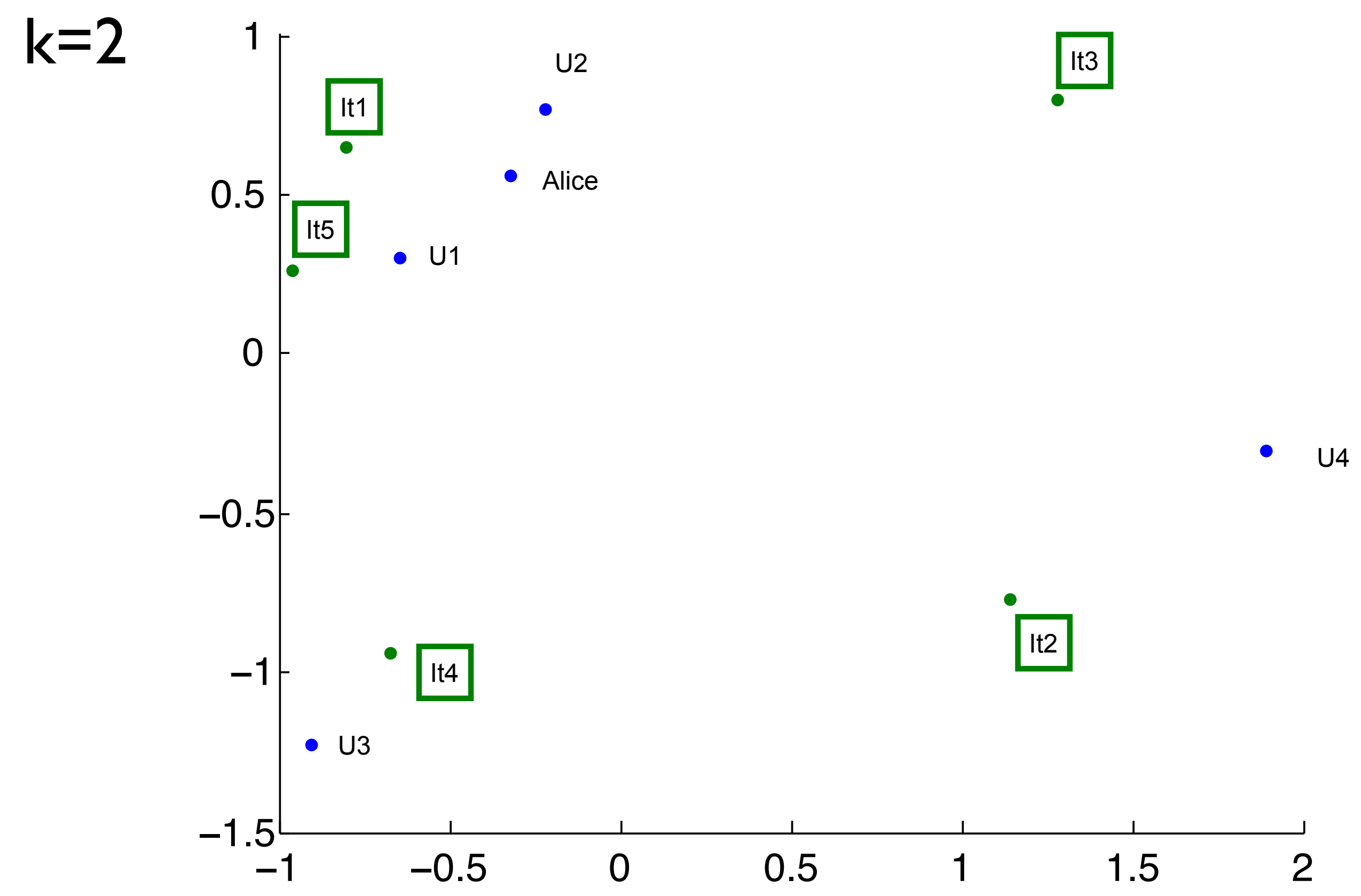




\section{Matrix factorization}

For $\mathrm{k}=2$;

$\mathrm{M} 2=\mathrm{U} 2{ }^{*} \mathrm{~S} 2{ }^{*}(\text { Items } 2)^{\prime}$

prediction2 $=(\mu)-b_{u}-b_{i}+M 2(1,5)=4.4602$

mean_error $=$ mean $($ mean $(\operatorname{abs}(M-M 2)))=0.1933$

For $\mathrm{k}=3$;

$\mathrm{M} 3=\mathrm{U}^{*} \mathrm{~S} 3^{*}(\text { Items } 3)^{\prime}$

prediction3 $=(\mu)-b_{u}-b_{i}+M 3(1,5)=4.0356$

mean_error $=$ mean $($ mean $($ abs $(M-M 3)))=0.0624$ 


\section{Matrix factorization}

However,

svd needs full matrices.

Earlier works relied on imputation:

- increases enormously the amount of data to be handled

- data is distorted due to inaccurate imputations 


\section{Matrix factorization}

To compute all estimators, Koren and Bell, set an optimization problem that admits an efficient solution and avoids the problem of missing values

$$
\begin{gathered}
\min _{b_{*}, q_{*}, p_{*}} \sum_{(u, i) \in \mathscr{K}}\left(r_{u i}-\mu-b_{i}-b_{u}-q_{i}^{T} p_{u}\right)^{2}+\lambda_{4}\left(b_{i}^{2}+b_{u}^{2}+\left\|q_{i}\right\|^{2}+\left\|p_{u}\right\|^{2}\right) \\
\hat{r}_{u i}=\mu+b_{i}+b_{u}+q_{i}^{T} p_{u}
\end{gathered}
$$

$q_{i}$ and $p_{u}$ are vectors of $k$ components inspired in rows and columns of the svd full matrix approach 


\section{Matrix factorization}

The optimization problem can be solved

Alternating least squares technique rotate between

fixing the pu's to solve for the qi's, and

fixing the qis to solve for the pu's

(each are quadratic problems that can be optimally solved)

Stochastic Gradient Descent 


\section{Matrix factorization}

The optimization problem can be solved

Stochastic Gradient Descent

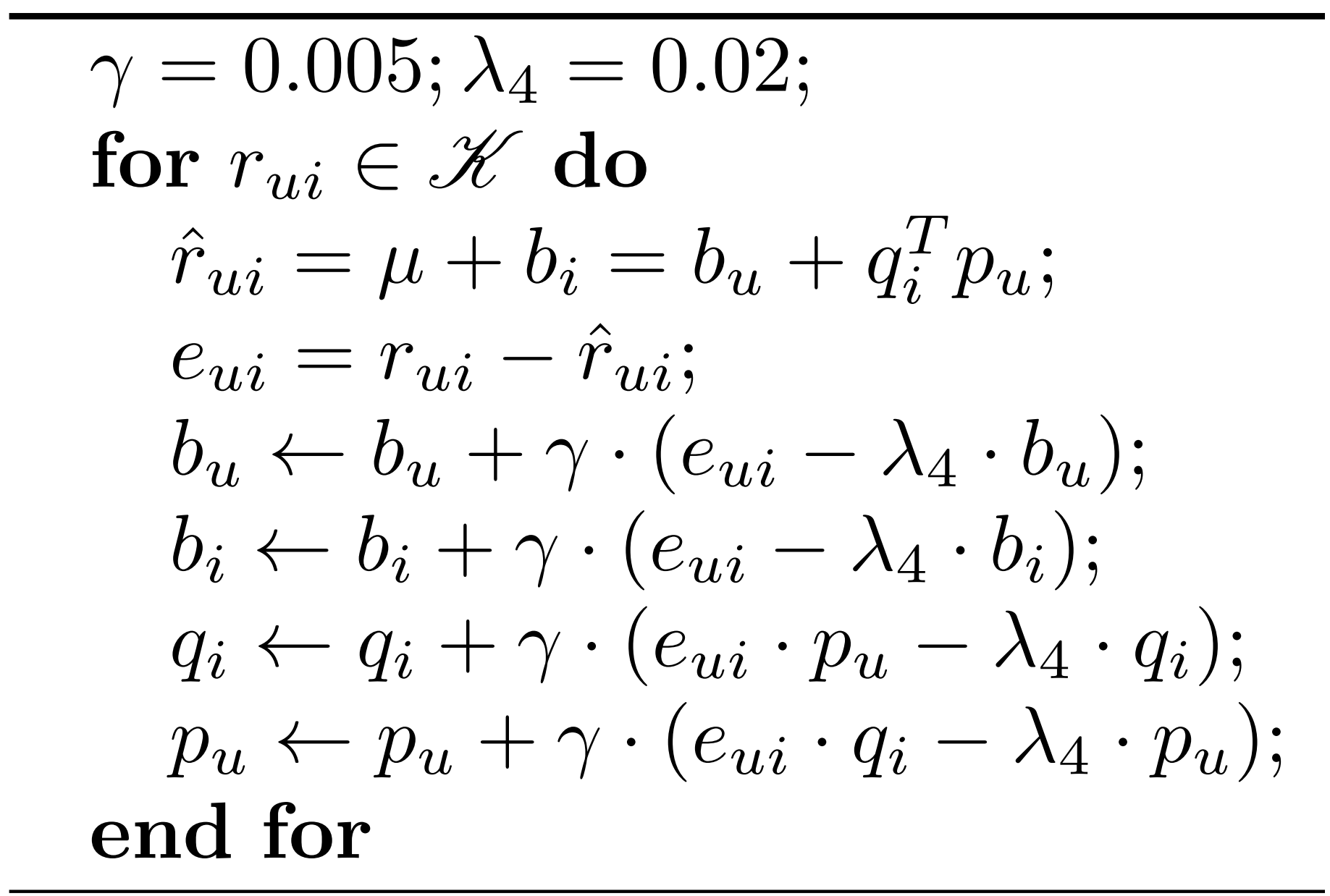




\section{Matrix factorization with implicit feedback}

Considering the set $R(u)$ of items that each user $u$ has rated as an implicit feedback

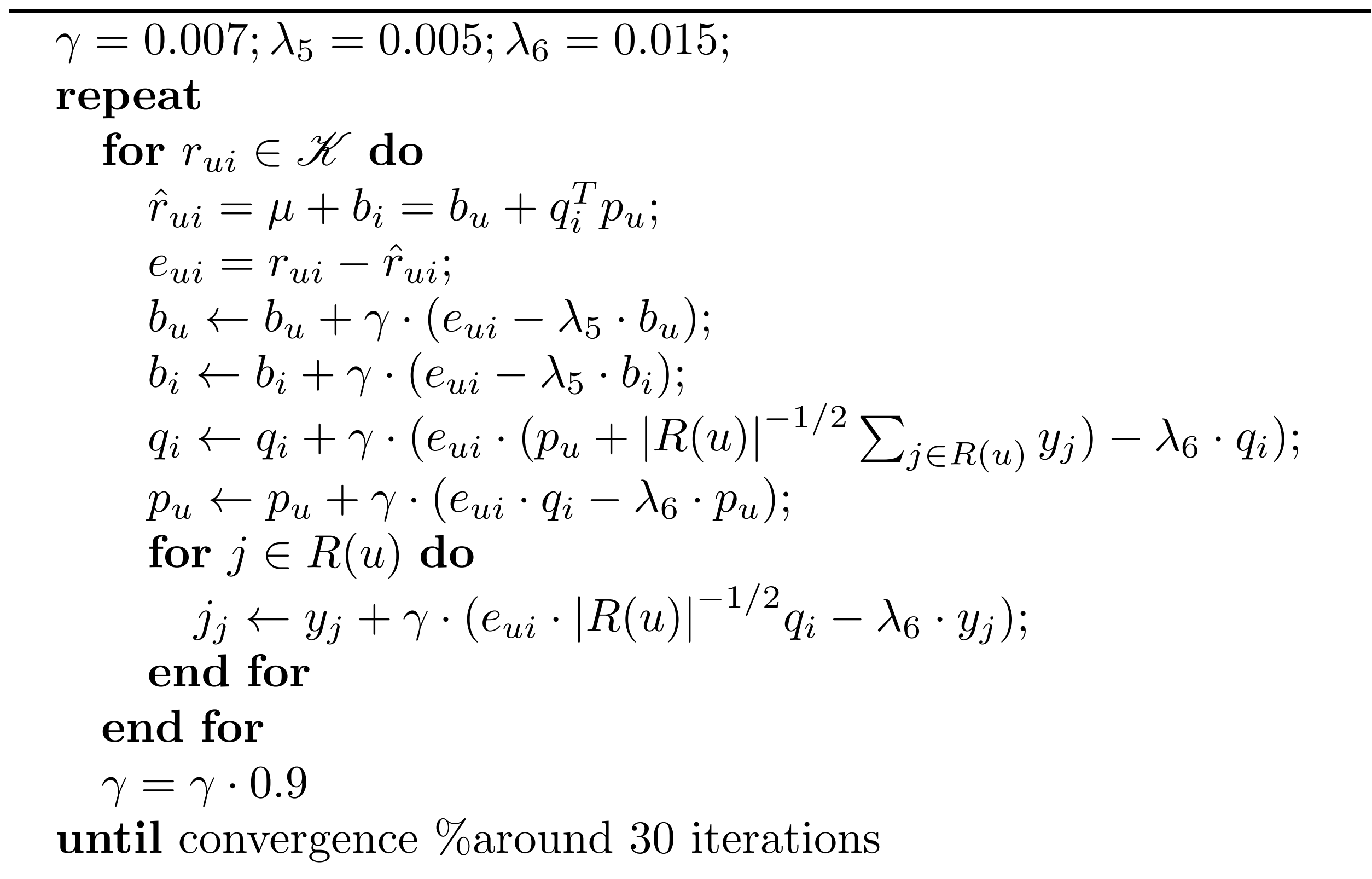




\section{Matrix factorization scores}

SVD method, with improvements in temporal drift and implicit feedback increases its performance. The value of the rank $k$ is also significant

\begin{tabular}{|c|c|c|c|c|c|}
\hline & $\mathbf{k}=\mathbf{1 0}$ & $\mathbf{k = 2 0}$ & $\mathbf{k = 5 0}$ & $\mathbf{k = 1 0 0}$ & $\mathbf{k = 2 0 0}$ \\
\hline SVD & 0,9140 & 0,9074 & 0,9046 & 0,9025 & 0,9009 \\
\hline SVD++ & 0,9131 & 0,9032 & 0,8952 & 0,8924 & 0,8911 \\
\hline times SVD++ & 0,8971 & 0,8891 & 0,8824 & 0,8805 & 0,8799 \\
\hline
\end{tabular}




\section{Matrix factorization scores}

Finally, with some extra improvements in the algorithms to solve the optimization problems, the team of Koren and Bell won the Netflix Prize with a

$$
\text { RMSE }=0.8567
$$

Remember that the second team (The Ensemble) reached the same score. The victory was awarded to Koren and Bell since their results were submitted 20 minutes before. 


\section{Other Recommender Systems}

\section{LME Music Embedding Demo}

(For best results, please use a Google Chrome browser.)

- Playlists:

Shuo Chen, Joshua Moore, Douglas Turnbull, Thorsten Joachims, Playlist Prediction via Metric Embedding, ACM Conference on Knowledge Discovery and Data Mining (KDD), 2012.

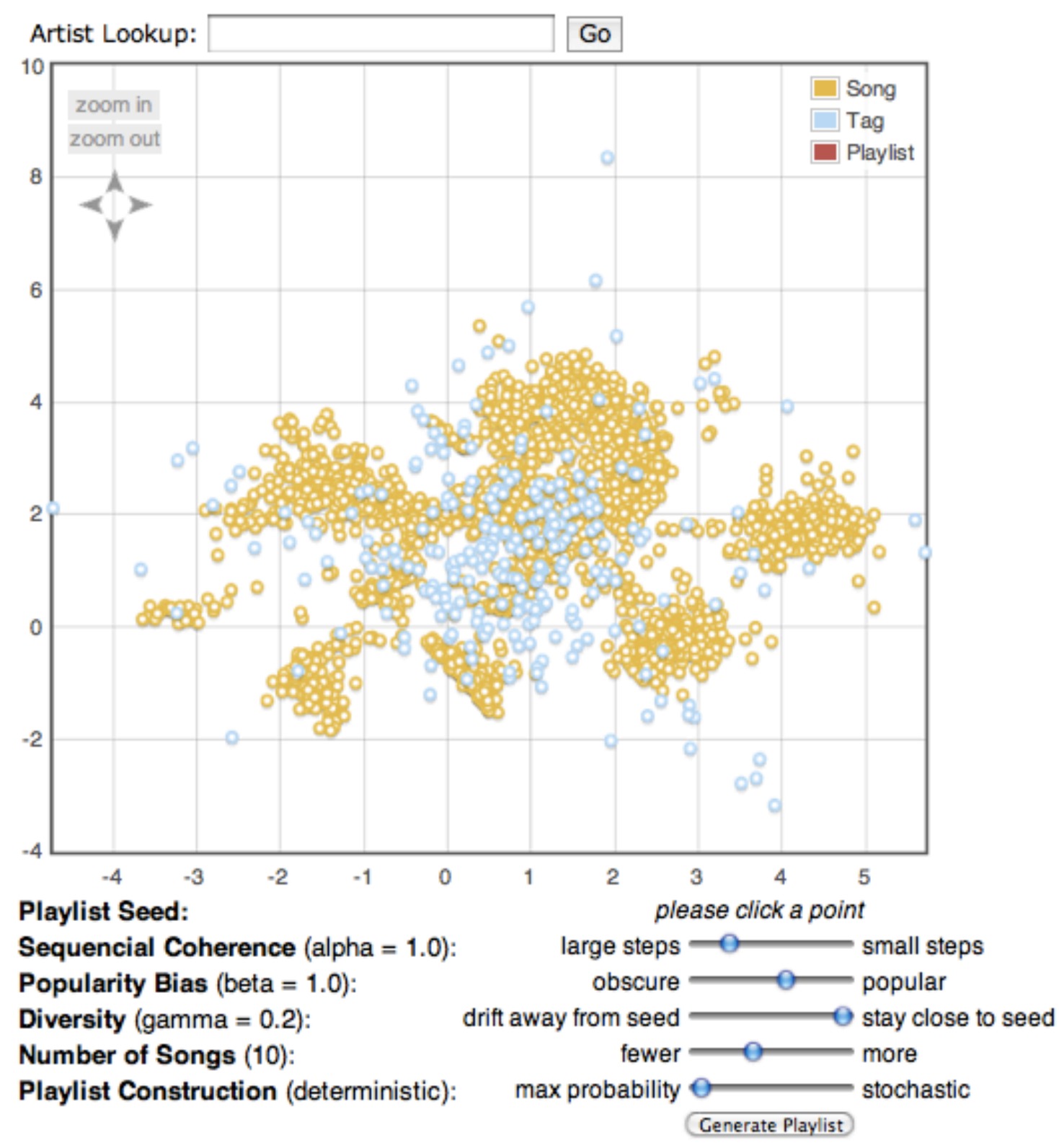




\section{References}

- Yehuda Koren, Robert M. Bell:Advances in Collaborative Filtering. Recommender Systems Handbook 20 I I: I45-I86

- William W. Cohen: Collaborative Filtering:A Tutorial. Carnegie Mellon University

- Dietmar Jannach, Gerhard Friedrich:Tutorial: Recommender Systems. International Joint Conference on Artificial Intelligence (IJCAI). Barcelona, July I7, 20 I I.TU Dortmund, Alpen-Adria Universität Klagenfurt) 